\title{
Modelos matemáticos no estudo do fluxo biológico de fósforo em ovinos que receberam na dieta níveis crescentes do mineral
}

\author{
[Mathematical models to study the biological phosphorus flow in sheep fed increasing levels of mineral] \\ R.M.P. Pardo ${ }^{1}$, T.S. Silva ${ }^{2}$, J.C. Silva Filho ${ }^{3}$, J.A. Moreira ${ }^{2}$, D.M.S.S. Vitti ${ }^{2}$ \\ ${ }^{1}$ Programa de Zootecnia - Universidade de Sucre, Cr. 28 No.5 - 267, Sincelejo, Colômbia \\ ${ }^{2}$ Centro de Energia Nuclear na Agricultura - USP - Piracicaba, SP \\ ${ }^{3}$ Programa de Zootecnia - UFLA - Lavras, MG
}

\begin{abstract}
RESUMO
Dois modelos compartimentais foram aplicados e comparados para avaliar o fluxo biológico de fósforo em ovinos que receberam dietas com níveis crescentes do mineral - 0, 2, 4 e 6 g por dia. Foram utilizados 24 machos, da raça Santa Inês, com média de peso de 33,6kg. Foi utilizado fosfato bicálcico como fonte de fósforo e ${ }^{32} \mathrm{P}$ como traçador. Avaliou-se o fluxo de fósforo entre os compartimentos: trato gastrintestinal, sangue, ossos e tecidos moles, além da ingestão, excreção e balanço do mineral. O incremento na ingestão de $\mathrm{P}$ aumentou a perda fecal do mineral. $\mathrm{O}$ fluxo de fósforo entre o trato gastrintestinal e o sangue e o fluxo contrário foram influenciados de forma quadrática pelo incremento na ingestão, diminuindo após a ingestão de 5,5g/dia, sem diferença entre os modelos avaliados. Os modelos estudados mostraram diferenças em relação ao fluxo entre sangue, ossos e tecidos moles, sem efeito dos tratamentos sobre o balanço geral do mineral, porém os níveis de ingestão praticados interferiram no fluxo biológico do fósforo. A disponibilidade biológica do fósforo ingerido diminuiu quando a ingestão superou a necessidade do animal, o que aumentou as excreções no ambiente. A quantificação do fluxo biológico de fósforo diferiu quando aplicados os modelos estudados em decorrência da sua estrutura.
\end{abstract}

Palavras-chave: ovino, Santa Inês, diluição isotópica, poluição ambiental

\begin{abstract}
Two compartimental models were applied and compared to evaluate the biological flow of $P$ in lambs fed diets containing increasing levels of $P(0,2,4$ and $6 \mathrm{~g}$ per day). Twenty four Santa Inês lambs with liveweight of $33.6 \mathrm{~kg}$ were used. Dicalcium phosphate was used as $P$ source and ${ }^{32} P$ as a tracer. $P$ flow between compartments (gastrointestinal tract, blood, bones and soft tissues), ingestion, excretion and the mineral balance were evaluated. The increase in $P$ intake increased fecal $P$ loss. $P$ flow from gastrointestinal tract to blood stream and opposite flow were affected quadratically by increased $P$ intake, decreasing after the ingestion of 5.5g/day, without a difference among models. The models studied showed differences regarding the $P$ flow between blood, bone and soft tissues, however, the $P$ balance was not affected by the treatments. The increased $P$ levels interfered with the biological $P$ flow in sheep. The bioavailability of $P$ diet decreases when intake exceeds the animal requirement, increasing losses to the environment. The quantification of biological $P$ flow was different between models due to the structure of each one.
\end{abstract}

Keywords: lamb, Santa Inês, isotopic dilution, environmental pollution 


\section{INTRODUÇÃO}

O P é considerado um elemento essencial para a vida. No animal, a maior parte do mineral (80\%) encontra-se nos ossos, e os $20 \%$ restantes participam do intenso metabolismo de energia, formando ATP, ADP e AMP. Está presente também nas moléculas de DNA e RNA e nas membranas citoplasmáticas como fosfolipídios.

Os ruminantes utilizam mais eficientemente o $\mathrm{P}$ dos vegetais em comparação aos não ruminantes, devido à síntese da enzima fitase pelos microrganismos do rúmen, o que não ocorre com os monogástricos. As pesquisas são direcionadas às deficiências do $\mathrm{P}$ nas pastagens, em função das deficiências em $\mathrm{P}$ dos solos brasileiros.

A quantidade excretada de P varia de acordo com a dieta, sendo a principal rota de excreção as fezes (Bravo et al., 2003). A quantidade e a fonte de $\mathrm{P}$ usadas na dieta podem afetar a cinética do mineral no organismo (Vitti et al., 2000; Dias et al., 2006). Também, a idade do animal pode ter influência na utilização do $\mathrm{P}$ dietético pela possível diferença na eficiência de absorção segundo a fase fisiológica (Braithwaite, 1975). Dessa maneira, são vários os aspectos que podem influenciar a homeostase de P no organismo e, por tanto, a cinética do mineral.

$\mathrm{O}$ P é considerado um agente poluente, que propicia o processo de eutroficação das águas. $\mathrm{O}$ foco de atenção de muitas pesquisas atuais tem sido a diminuição da excreção de $\mathrm{P}$ sem afetar o desempenho animal, por meio do correto balanceamento da dieta, para o qual é necessário conhecer as implicações metabólicas devido a modificações nos aspectos dietéticos relacionados ao $\mathrm{P}$.

A compreensão do metabolismo de $\mathrm{P}$ em ruminantes tem melhorado devido à utilização da técnica da diluição isotópica e à aplicação de modelos que representam a cinética do $P$. Fernandez (1995), ao utilizar dados combinados de estudos de balanço e absorção de $\mathrm{P}$ com estudos de cinética em suínos, formulou um modelo de três compartimentos (trato gastrintestinal, plasma e osso) para estudar o metabolismo de Ca e P. Lopes et al. (2001) adaptaram o modelo anterior e acrescentaram um novo compartimento para representar os tecidos. Vitti et al. (2000) propuseram, também, um modelo compartimental (trato gastrintestinal, plasma, ossos e tecidos moles) para estudar a cinética de $\mathrm{P}$ usando fósforo marcado $\left({ }^{32} \mathrm{P}\right)$ e aplicando o princípio de conservação das massas para gerar equações diferenciais que descrevem a dinâmica do sistema.

Os níveis de ingestão utilizados neste estudo justificam-se pelos poucos estudos que avaliam níveis de ingestão de até $220 \mathrm{mg} / \mathrm{kg} \mathrm{PV}$ em ovinos com peso próximo de $35 \mathrm{~kg}$.

O experimento foi realizado com o objetivo de estudar o efeito da ingestão de $\mathrm{P}$ sobre o fluxo biológico de $\mathrm{P}$ em ovinos em crescimento, comparando dois modelos matemáticos de tipo compartimental.

\section{MATERIAL E MÉTODOS}

O experimento foi realizado no Laboratório de Nutrição Animal do Centro de Energia Nuclear na Agricultura (CENA), da Universidade de São Paulo. Foram utilizados 24 ovinos machos inteiros da raça Santa Inês, com peso de 33,6kg $( \pm 1,6)$. Foram adotados dois períodos experimentais. Os animais foram mantidos, inicialmente, em baias de alvenaria, durante sete dias, onde começaram a receber as dietas experimentais. Posteriormente, os cordeiros foram transferidos para gaiolas de estudo de metabolismo, equipadas com coletores para fezes e urina, cochos de alimentação e bebedouro, onde permaneceram por duas semanas, sendo a primeira semana a de adaptação.

Os tratamentos consistiram em quantidades crescentes de P suplementar na dieta - 0; 2; 4 e $6 \mathrm{~g} /$ dia. Excluindo a dieta do tratamento sem suplementação de P (dieta basal), constituída por $300 \mathrm{~g} /$ dia de concentrado e feno de capim coastcross (Cynodum dactylon L. pers) à vontade, às demais foi adicionado o fosfato bicálcico (16\% de $\mathrm{P}$ ) para atingir os níveis propostos de $\mathrm{P}$ suplementar (Tab. 1). As dietas foram isoenergéticas e isoproteicas, e com relação Ca:P semelhante. A dieta base foi formulada para atender às exigências nutricionais para esse tipo de animal, utilizando-se as recomendações do NRC (Nutrient..., 2007), e as demais para prover teores de $\mathrm{P}$ e $\mathrm{Ca}$ superiores às exigências. $\mathrm{O}$ concentrado, com o fosfato, e o feno foram oferecidos em cochos separados. O concentrado, que não apresentou sobras, foi oferecido de 
manhã (8h) e o feno duas vezes ao dia (8 e 17h), para permitir $10 \%$ de sobras. O consumo de alimento e água foi calculado e anotado diariamente.

Amostras dos ingredientes e das sobras da ração foram coletadas para a determinação de matéria seca, matéria mineral, extrato etéreo e proteína bruta, seguindo a metodologia da AOAC (Official..., 1995). A fibra em detergente neutro (FDN) e a fibra em detergente ácido (FDA) foram determinadas segundo Van Soest e Wine (1967). A determinação de $P$ foi feita por colorimetria (Sarrugue e Haag, 1974) e a de Ca por espectrofotometria de absorção atômica (Zagatto et al., 1979).

Tabela 1. Constituição e composição bromatológica das dietas nos diferentes níveis de suplementação de $\mathrm{P}$

\begin{tabular}{lcccc}
\hline \multirow{2}{*}{ Constituinte $^{1}$, \% } & \multicolumn{3}{c}{ P suplementar (g/dia) } \\
\cline { 2 - 4 } & 0 & 2 & 4 & 6 \\
\hline Feno de coast cross & 71,6 & 70,6 & 69,7 & 68,8 \\
Farinha de mandioca & 18,8 & 18,5 & 18,3 & 18,1 \\
Farelo de soja & 5,7 & 5,6 & 5,6 & 5,5 \\
Melaço & 1,2 & 1,2 & 1,2 & 1,2 \\
Ureia & 1,7 & 1,7 & 1,7 & 1,6 \\
Fosfato bicálcico & - & 1,3 & 2,6 & 3,9 \\
Mistura mineral* & 1,0 & 1,0 & 1,0 & 1,0 \\
\hline Composição ${ }^{2}, \%$ & & & 49,6 & 49,0 \\
\hline NDT & 50,9 & 50,3 & 11,4 \\
Proteína bruta & 11,9 & 11,7 & 1,6 \\
Extrato etéreo & 1,7 & 1,6 & 1,6 & 10,8 \\
Matéria mineral & 7,2 & 8,5 & 9,7 & 57,0 \\
FDN & 59,3 & 58,6 & 57,8 & 31,3 \\
FDA & 32,6 & 32,2 & 31,7 & 0,79 \\
P & 0,16 & 0,37 & 0,58 & 1,64 \\
Ca & 0,35 & 0,78 & 1,22 & 2,1 \\
Ca:P & 2,2 & 2,1 & 2,1 & \\
\hline
\end{tabular}

${ }^{1}$ Na matéria natural.

${ }^{2} \mathrm{Na}$ matéria seca.

*Composição: Cl, 219mg/g; Na, 145mg/g; S, 70mg/g; Mg, 10mg/g; Zn, 4,6mg/g; Mn, 1,1mg/g; Fe, 0,50mg/g; Cu, 0,30mg/g; I, 80mg/kg; Co, 40mg/kg; e Se, $15 \mathrm{mg} / \mathrm{kg}$.

No oitavo dia nas gaiolas, $7,4 \mathrm{MBq}$ de ${ }^{32} \mathrm{P}$, em $1 \mathrm{~mL}$ de solução salina $0,85 \%$, foram injetados em cada animal, na jugular direita. Para o padrão, foi utilizado $0,5 \mathrm{~mL}$ dessa solução, e a radioatividade medida por meio do efeito Cerenkov (Nascimento Filho e Lobão, 1977) em espectrofotômetro de cintilação líquida (Beckman LS 5000 - TA), como foi feito também para as amostras de plasma, fezes, urina e tecidos. Os períodos de colheitas de sangue foram cinco minutos após a injeção, e a cada 24 horas, durante sete dias. $\mathrm{O}$ sangue foi centrifugado a $1100 \times g$ por 10 minutos, para separar o plasma. Amostras de $1 \mathrm{~mL}$ de plasma foram misturadas a $9 \mathrm{~mL}$ de ácido tricloroacético (10\%) para precipitação das proteínas. Após 10 minutos, o material foi centrifugado a $1100 \times \mathrm{g}$, e o sobrenadante separado para a determinação dos teores de P inorgânico por colorimetria (Fiske e Subbarow, 1925).

Diariamente, após pesagem e homogeneização das fezes, alíquotas correspondentes a 10\% do total diário excretado eram maceradas, homogeneizadas e congeladas $\left(-20^{\circ} \mathrm{C}\right)$. Alíquotas de $1 \mathrm{~g}$ foram queimadas em forno mufla $\left(500^{\circ} \mathrm{C}\right)$, e as cinzas digeridas com $5 \mathrm{~mL}$ de ácido clorídrico concentrado. O teor de $\mathrm{P}$ foi, então, determinado por colorimetria (Sarruge e Haag, 1974). A porcentagem de $P$ de origem endógena nas fezes e a disponibilidade biológica do $\mathrm{P}$ foram calculadas segundo Portilho et al. (2006), utilizando a técnica da diluição isotópica.

A urina foi coletada em frascos contendo $100 \mathrm{~mL}$ de ácido sulfúrico a $10 \%$ e alíquotas correspondentes a $10 \%$ do total diário foram 
armazenadas. Os teores de P inorgânico na urina foram determinados por colorimetria (Fiske e Subbarow, 1925). Os cordeiros foram sacrificados 168 horas após a injeção do material radioativo, de acordo com os procedimentos da Comissão de Ética em Experimentação com Animais do CENA. Após a sangria dos animais, fígado, coração, rins e testículos foram separados e pesados. Uma amostra de tecido muscular foi obtida realizando-se um corte transversal, de aproximadamente $5 \mathrm{~cm}$ de largura, no membro posterior direito. A amostra de osso foi representada pela $12^{\mathrm{a}}$ costela do lado direito do animal. O restante da carcaça foi levado para um depósito construído sob supervisão do Serviço de Proteção Radiológica do CENA. As amostras dos tecidos foram limpas e preparadas para a determinação do teor de matéria seca, seguindose uma pré-queima com ácido nítrico $50 \%$. As cinzas obtidas por incineração $\left(500^{\circ} \mathrm{C}\right)$ foram solubilizadas em ácido clorídrico, e o teor de fósforo inorgânico determinado pelo método vanadato-molibdato (Sarruge e Haag, 1974).

O modelo 1 (Fig. 1), utilizado para estudar a cinética de $\mathrm{P}$, foi o inicialmente proposto por Vitti et al. (2000). Os símbolos usados no modelo de tipo compartimental foram: $F_{i j}=$ fluxo total de $\mathrm{P}$ para $i$ a partir do $j, \mathrm{Fi}_{0}$ denota fluxo externo dentro de $i$ e $F_{0 j}$ o fluxo de saída do sistema a partir de $j$, os fluxos $F_{01}, F_{10}$ e $F_{02}$ foram medidos experimentalmente: $\mathrm{D}=$ dose de ${ }^{32} \mathrm{P}$ administrada no sangue no tempo zero em cpm; $\mathrm{Q}_{\mathrm{i}}=$ quantidade total de $\mathrm{P}$ em $i ; \mathrm{q}_{\mathrm{i}}=$ quantidade de ${ }^{32} \mathrm{P}$ em $i$ em com; $\mathrm{s}_{\mathrm{i}}=$ atividade específica do pool i $\left(\mathrm{q}_{\mathrm{i}} / \mathrm{Q}_{\mathrm{i}}\right)$ em cpm/g; $\mathrm{T}=$ tempo (dia). A unidade cpm correspondeu à contagem ou decaimento por minuto. O modelo possui quatro compartimentos: trato digestivo (1), plasma (2), ossos (3) e tecidos moles (4). Está representado por setas o fluxo do $\mathrm{P}$ entre os compartimentos, assim como as entradas e as saídas do sistema. O trato gastrintestinal, ossos e tecidos moles apresentam trocas bidirecionais com o plasma. O P entra no sistema via consumo $\left(F_{10}\right)$ e sai através das fezes $\left(F_{01}\right)$ e urina $\left(F_{02}\right)$. O radioisótopo $\left({ }^{32} \mathrm{P}\right)$ foi administrado como uma dose única (D, cpm), no tempo zero, e o tamanho do compartimento, que correspondeu à quantidade de $\mathrm{P}$ de cada compartimento, bem como a atividade específica no plasma, os ossos e os tecidos moles foram medidos após oito dias. O esquema assume que não há reentrada de marcador de outras fontes externas.

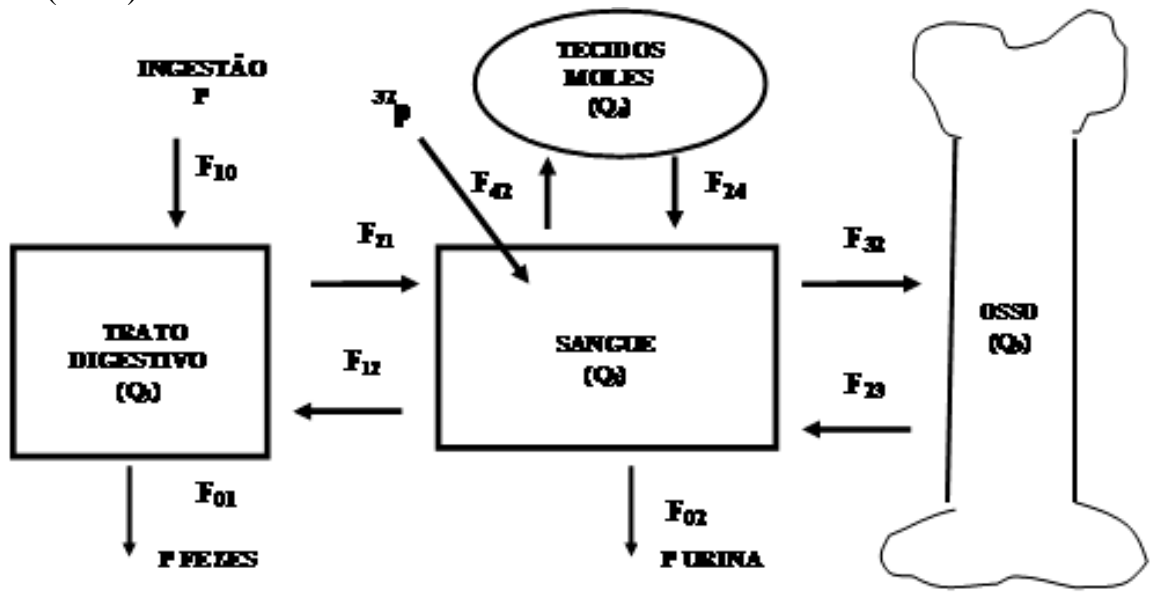

Figura 1. Modelo de Vitti et al. (2000) para distribuição de P no metabolismo de ovinos.

Após sete dias da introdução do ${ }^{32} \mathrm{P}$, assume-se que o compartimento 1 está em equilíbrio (isotópico e não isotópico), e o compartimento 2 em estado não isotópico. A solução algébrica do modelo foi representada pelas seguintes equações:

$$
\begin{aligned}
& F_{12}=s_{1} \tilde{F}_{10} /\left(s_{2}-s_{1}\right) \\
& F_{21}=\tilde{F}_{10}+F_{12}-\tilde{F}_{02} \\
& F_{32}=s_{3} R_{2} Q_{3} /\left[s_{2}\left(s_{2}-s_{3}\right)\right] \\
& F_{42}=s_{4} R_{2} Q_{4} /\left[s_{2}\left(s_{2}-s_{4}\right)\right]
\end{aligned}
$$

$$
\begin{aligned}
& \left|F_{23}+F_{24}\right|=\tilde{F}_{02}+F_{12}+F_{32}+F_{42}-F_{21} \\
& F_{24}=\left(s^{*}-s_{3}\right) \times\left|F_{23}+F_{24}\right| /\left(s_{4}-s^{*}\right) \\
& F_{23}=\left|F_{23}+F_{24}\right|-F_{24}
\end{aligned}
$$

em que a notação $\tilde{F}$ indica um fluxo determinado experimentalmente, e a atividade específica da combinação dos compartimentos 3 e 4 foi: $\mathrm{s}^{*}=\left(s_{3} Q_{3}+s_{4} Q_{4}\right) /\left(Q_{3}+Q_{4}\right)$ 
A taxa de troca da atividade específica do compartimento 2 foi:

$R_{2}=\mathrm{d} s_{2} / \mathrm{d} t$

Os valores de $s_{2}$ e $R_{2}$ aos sete dias foram obtidos após o ajuste da equação da curva de decaimento da atividade específica do plasma:

$s_{2}(t)=a e^{-b t}$

em que $t$ (dia) é o tempo desde a aplicação da dose , e $a(\mathrm{dpm} / \mathrm{g})$ e $\mathrm{t}$ (dia) são parâmetros. A taxa de troca resultante quando se diferencia a equação em relação ao tempo é igual a: $d s_{2}(t) / d t=-a b e^{-b t}$

a qual permite o cálculo de $R_{2}$ para o sétimo dia. O modelo 2 (Fig. 2) correspondeu ao proposto por Lopes et al. (2001). As trocas bidirecionais entre o plasma e os demais compartimentos foram representadas pelas variáveis $V_{\mathrm{aT}}, V_{\mathrm{IT}}, V_{\mathrm{o}^{+}}$, $V_{\text {o-, }}, V_{\mathrm{Te}^{+}}$e $V_{\mathrm{Te}-\text {. }}$ (Tab. 2).

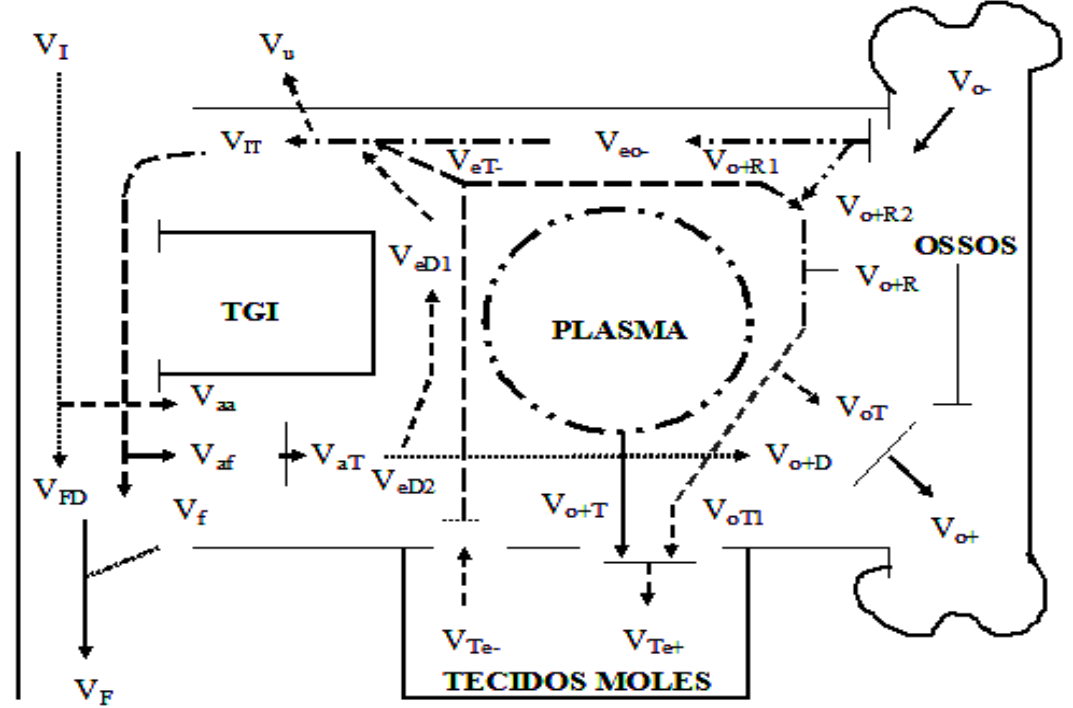

Figura 2. Esquema do modelo de metabolismo de fósforo de acordo com Lopes et al. (2001).

Os fluxos foram computados $\left(V_{\mathrm{F}}, V_{\mathrm{f}}, \mathrm{V}_{\mathrm{I}}\right.$, e $V_{\mathrm{u}}$ medidos experimentalmente) usando-se as seguintes equações:

$V_{\text {aa }}=V_{\mathrm{I}}-\left(V_{\mathrm{F}}-V_{\mathrm{f}}\right)$

$V_{\mathrm{IT}}=V_{\mathrm{f}} / 1-\left(V_{\mathrm{aa}} / V_{\mathrm{f}}\right)$

$V_{\mathrm{aT}}=V_{\text {aa }}+V_{\mathrm{IT}}-V_{\mathrm{f}}$

Balanço de $\mathrm{P}(\mathrm{Ba})=V_{\mathrm{I}}-V_{\mathrm{f}}-V_{\mathrm{u}}$
$V_{0^{+}}=$atividade acumulada nos ossos/atividade específica no plasma

$V_{\mathrm{o}^{-}}=V_{\mathrm{o}^{+}}-\mathrm{Ba}$

$V_{\mathrm{te}^{+}}=$atividade acumulada nos tecidos/atividade específica no plasma

$V_{\text {te- }}=V_{\text {te }}-$ Ba

Tabela 2. Variáveis relacionadas ao metabolismo de P do modelo de Lopes et al. (2001)

\begin{tabular}{ll}
\hline$V_{\mathrm{I}}$ & P ingerido \\
$V_{\mathrm{F}}$ & P total nas fezes \\
$V_{\mathrm{f}}$ & P endógeno fecal \\
$V_{\mathrm{u}}$ & P na urina \\
$V_{\mathrm{aa}}$ & P absorvido de origem alimentar \\
$V_{\mathrm{IT}}$ & P de origem endógena que chega ao trato gastrintestinal \\
$V_{\mathrm{af}}$ & P endógeno reabsorvido no trato gastrintestinal \\
$V_{\mathrm{aT}}$ & P total absorvido \\
$V_{\mathrm{O}+}$ & P incorporado no osso \\
$V_{\mathrm{o}-}$ & P reabsorvido do osso \\
$V_{\mathrm{eo}-}$ & P reabsorvido do osso que retorna ao trato gastrintestinal \\
$V_{\mathrm{o}+\mathrm{D}}$ & P do $V_{\mathrm{aT}}$ incorporado no osso \\
$V_{\mathrm{Te}+}$ & P incorporado nos tecidos moles \\
$V_{\mathrm{Te}-}$ & P reabsorvido dos tecidos moles \\
\hline
\end{tabular}


Utilizou-se delineamento em blocos ao acaso, com três repetições por tratamento dentro de cada bloco, para um total de seis repetições por tratamento. Foi realizada análise de variância com o modelo considerando os efeitos das fontes de variação: grupo (como bloco); teor de $\mathrm{P}$ da dieta, com soma dos quadrados dividida para testar os efeitos linear, quadrático e cúbico, com 1 grau de liberdade para cada contraste, e o erro. A comparação dos valores médios das variáveis comuns para os dois modelos foi realizada usando-se um teste $t$ a $5 \%$ de probabilidade. A análise estatística dos dados foi realizada usandose as funções comparação de médias, GLM e correlação do programa SPSS ${ }^{\circledR}$ (Statistical..., 2006) versão 15 para Windows. Valores de probabilidade entre 0,05 e 0,15 para o fluxo $F_{02}$ foram considerados como uma tendência.

\section{RESULTADOS E DISCUSSÃO}

$\mathrm{O}$ teor de $\mathrm{P}$ da dieta influiu de maneira linear $(\mathrm{P}<0,001)$ na ingestão do mineral (Tab. 3). Houve também efeito linear $(\mathrm{P}<0,001)$ positivo do teor de $\mathrm{P}$ da dieta sobre a excreção fecal de $\mathrm{P}$ $\left(F_{01}, V_{\mathrm{F}}\right)$. A associação entre a ingestão de $\mathrm{P}$ e a excreção fecal de $\mathrm{P}$ foi explicada pela equação: $F_{01}=0,88 F_{10}-0,14\left(r^{2}=0,95, \mathrm{P}<0,001\right)$. Relações semelhantes foram observadas em trabalhos anteriores (Louvandini e Vitti, 2007; Dias et al., 2007) em ovinos.

Tabela 3. Consumo e excreção de P, atividades específicas (ATE) e teores de P no plasma, nas fezes e nos ossos de ovinos suplementados com diferentes quantidades de $\mathrm{P}$

\begin{tabular}{|c|c|c|c|c|c|}
\hline \multirow{2}{*}{ Variável } & \multicolumn{4}{|c|}{ P suplementar (g/dia) } & \multirow{2}{*}{$\mathrm{EPM}^{1}$} \\
\hline & 0 & 2 & 4 & 6 & \\
\hline \multicolumn{6}{|l|}{ Fluxos de P (g/dia) } \\
\hline Consumo $\left(F_{10}, V_{\mathrm{I}}\right)$ & 1,47 & 3,53 & 5,52 & 7,47 & 0,21 \\
\hline Fezes $\left(F_{01}, V_{\mathrm{F}}\right)$ & 1,09 & 3,18 & 4,40 & 6,55 & 0,09 \\
\hline Urina $\left(F_{02}, V_{\mathrm{u}}\right)$ & 0,003 & 0,01 & 0,07 & 0,36 & 0,08 \\
\hline $\mathrm{P}$ da dieta absorvido & 0,98 & 1,77 & 3,53 & 3,36 & 0,10 \\
\hline P endógeno absorvido & 1,32 & 1,59 & 5,25 & 2,25 & 0,39 \\
\hline \multicolumn{6}{|l|}{ ATE $(\mathrm{cpm} / \mathrm{gP})$} \\
\hline Fezes $\left(s_{1}\right)$ & 13,93 & 8,42 & 6,45 & 3,72 & 0,83 \\
\hline Plasma $\left(s_{2}\right)$ & 26,63 & 17,12 & 12,12 & 10,23 & 1,48 \\
\hline Ossos $\left(s_{3}\right)$ & 3,33 & 1,95 & 2,23 & 1,73 & 0,17 \\
\hline Tecidos $\left(s_{4}\right)$ & 6,53 & 4,75 & 3,43 & 3,10 & 0,37 \\
\hline \multicolumn{6}{|l|}{ Quantidade de P (g) } \\
\hline Plasma $\left(Q_{2}\right)$ & 0,10 & 0,13 & 0,14 & 0,13 & 0,06 \\
\hline Osso $\left(Q_{3}\right)$ & 147,4 & 148,9 & 149,2 & 149,9 & 3,24 \\
\hline Tecidos moles $\left(Q_{4}\right)$ & 18,90 & 18,64 & 21,61 & 21,26 & 0,75 \\
\hline
\end{tabular}

${ }^{1}$ EPM: erro-padrão da média.

Com relação à excreção de P pela urina (Tab. 3), foi observado efeito linear $(\mathrm{P}=0,11)$ de aumento da excreção de $\mathrm{P}$ quando se aumentou a ingestão. A urina representou 0,$27 ; 0,31 ; 1,57$ e $5,2 \%$ do total de $\mathrm{P}$ excretado, nos níveis de suplementação de 0, 2, 4 e $6 g$ por dia, respectivamente. Portanto, as fezes representaram a principal saída de $\mathrm{P}$ do sistema (>90\% da excreção total).

Efeito quadrático $(\mathrm{P}<0,025)$ foi observado na atividade específica em fezes e plasma, diferente das atividades nos ossos e tecidos moles, que apresentaram efeito linear $(\mathrm{P}<0,001)$ do incremento da ingestão sobre estas variáveis (Tab. 3). Os resultados são coerentes com a afirmação de Kleiber et al. (1951) no sentido de que, à medida que se aumenta a quantidade de $\mathrm{P}$ da dieta presente nas fezes, o $\mathrm{P}$ radiativo é diluído, portanto os valores da atividade específica diminuem. Dias et al. (2007) explicaram o efeito quadrático da ingestão de $\mathrm{P}$ sobre a atividade específica no plasma, indicando que a atividade no plasma não varia quando são ingeridas grandes quantidades de $\mathrm{P}$.

A quantidade de $\mathrm{P}$ no plasma e tecidos moles foi afetada linearmente $(\mathrm{P}<0,03)$ com o aumento na ingestão de $\mathrm{P}$, não sendo observado efeito sobre a quantidade de $\mathrm{P}$ nos ossos $(\mathrm{P}>0,812)$. Dias et al. (2007) não observaram efeitos do incremento na ingestão de $\mathrm{P}$ sobre a quantidade de $\mathrm{P}$ no plasma, ossos e tecidos. 
Os fluxos $F_{21}\left(V_{\mathrm{aT}}\right)$ e $F_{12}\left(V_{\mathrm{IT}}\right)$, que representam os fluxos de $\mathrm{P}$ entre TGI e plasma e vice-versa, respectivamente, foram afetados de forma quadrática $(\mathrm{P}<0,03)$ pelo incremento na ingestão de $\mathrm{P}$ (Tab. 4) e apresentaram correlação significativa $\left(r^{2}=0,98 ; \mathrm{P}<0,001\right)$.

Tabela 4. Médias dos fluxos de P em ovinos suplementados com diferentes quantidades de P, de acordo com os modelos Vitti et al. (2000) e Lopes et al. (2001)

\begin{tabular}{|c|c|c|c|c|c|}
\hline \multirow{2}{*}{ Fluxos ${ }^{1,2}$ (Símbolo) } & \multicolumn{4}{|c|}{ P suplementar (g/dia) } & \multirow{2}{*}{$\mathrm{EPM}^{3}$} \\
\hline & 0 & 2 & 4 & 6 & \\
\hline \multicolumn{6}{|l|}{ Modelo $1^{*}$ (gP/dia) } \\
\hline TGI - Plasma $\left(F_{21}\right)$ & 2,16 & 4,29 & 8,68 & 5,60 & 0,47 \\
\hline Plasma - TGI $\left(F_{12}\right)$ & 1,80 & 3,93 & 7,56 & 4,67 & 0,44 \\
\hline Plasma - Osso $\left(F_{32}\right)$ & 3,60 & 4,11 & 8,90 & 9,59 & 0,94 \\
\hline Osso - Plasma $\left(F_{23}\right)$ & 3,81 & 4,70 & 8,68 & 9,92 & 0,95 \\
\hline Plasma - TM $\left(F_{42}\right)$ & 1,06 & 1,50 & 2,11 & 2,43 & 0,18 \\
\hline TM - Plasma $\left(F_{24}\right)$ & 0,48 & 0,58 & 1,28 & 1,52 & 0,15 \\
\hline Balanço osso $\left(F_{32}-F_{23}\right)$ & $-0,21$ & $-0,59$ & 0,22 & $-0,33$ & 0,17 \\
\hline Balanço TM $\left(F_{42}-F_{24}\right)$ & 0,58 & 0,92 & 0,84 & 0,90 & 0,09 \\
\hline \multicolumn{6}{|l|}{ Modelo $2^{* *}$ (gP/dia) } \\
\hline TGI - Plasma $\left(V_{\mathrm{aT}}\right)$ & 2,30 & 3,36 & 8,78 & 5,61 & 0,48 \\
\hline Plasma - TGI ( $\left.V_{\text {IT }}\right)$ & 1,92 & 3,01 & 7,66 & 4,68 & 0,46 \\
\hline Plasma - Osso $\left(V_{\mathrm{o}^{+}}\right)$ & 6,34 & 6,38 & 6,20 & 6,21 & 0,126 \\
\hline Osso - Plasma ( $\left.V_{\mathrm{o}-}\right)$ & 5,99 & 6,37 & 5,21 & 5,67 & 0,213 \\
\hline Plasma - TM $\left(V_{\mathrm{Te}^{+}}\right)$ & 0,37 & 0,31 & 0,40 & 0,36 & 0,025 \\
\hline TM - Plasma $\left(V_{\text {Te- }}\right)$ & 0,35 & 0,31 & 0,33 & 0,39 & 0,024 \\
\hline Balanço osso $\left(V_{\mathrm{o}^{+}}-V_{\mathrm{o}_{-}}\right)$ & 0,35 & 0,01 & 0,99 & 0,54 & 0,117 \\
\hline Balanço TM $\left(V_{\mathrm{Te}+}-V_{\mathrm{Te}-}\right)$ & 0,02 & 0,0 & 0,06 & $-0,03$ & 0,008 \\
\hline
\end{tabular}

${ }^{1}$ TGI=trato gastrintestinal

${ }^{2} \mathrm{TM}=$ tecidos moles

${ }^{3}$ EPM: erro-padrão da média.

*Modelo Vitti et al. (2000).

${ }^{* *}$ Modelo de Lopes et al. (2001).

Após a ingestão de $5,52 \mathrm{~g}$ de $\mathrm{P}$ por dia $(0,58 \%$ de $\mathrm{P}$ na MS), os fluxos $F_{21}\left(V_{\mathrm{aT}}\right)$ e $F_{12}\left(V_{\mathrm{IT}}\right)$ diminuíram, indicando que, na maior ingestão de P (7,47 g/animal/dia ou 220mg/kg PV), houve inibição na absorção intestinal do elemento. Do P consumido, 37 a $55 \%$ foram excretados via $V_{\text {IT }}$ ou $F_{12}$. A menor disponibilidade biológica (45\%), na maior ingestão, coincidiu com a queda no fluxo entre TGI e plasma $\left(F_{21}\right.$ ou $\left.V_{\mathrm{aT}}\right)$, neste tratamento. Os demais tratamentos apresentaram disponibilidade biológica entre 55 e 66\%.

Dias et al. (2007) observaram efeito linear dos tratamentos sobre o fluxo de $\mathrm{P}$ de TGI para plasma ( $F_{21}$ ou $\left.V_{\mathrm{aT}}\right)$, com níveis de ingestão entre 39,5 e 286,5mg de $\mathrm{P} / \mathrm{kg}$ de PV, faixa mais ampla que a usada no presente trabalho, em que o nível máximo foi de $220,8 \mathrm{mg} / \mathrm{kg}$ de $\mathrm{PV}$, mas em animais com menor peso (23kg). Braithwaite (1975) também observou maior eficiência de absorção em animais jovens.
Quando comparados os valores médios dos fluxos entre TGI e plasma e vice-versa ( $F_{21} v s$. $V_{\mathrm{aT}}$ e $F_{12}$ vs. $\left.V_{\mathrm{IT}}\right)$, resultantes da aplicação dos dois modelos (Tab. 5), não foram verificadas diferenças significativas $(\mathrm{P}>0,05)$.

Aplicando-se o modelo 1, observou-se que os fluxos de $\mathrm{P}$ entre plasma e ossos ou tecidos moles e vice-versa $\left(F_{32}\right.$ e $F_{23} ; F_{42}$ e $\left.F_{24}\right)$ foram influenciados pelos tratamentos (Tab. 4). O incremento na ingestão de $\mathrm{P}$ resultou em aumento linear dos fluxos $F_{32}, F_{23}, F_{42}$ e $F_{24}$ $(\mathrm{P}<0,02)$. Foi verificada uma correlação significativa $\left(r^{2}=0,98 ; \mathrm{P}<0,001\right)$ entre os fluxos $F_{32}$ e $F_{23}$, e entre $F_{42}$ e $F_{24}\left(r^{2}=0,87 ; \mathrm{P}<0,001\right)$. Mas, quando aplicado o modelo 2, os fluxos equivalentes ao do modelo $1\left(V_{\mathrm{o}^{+}}, V_{\mathrm{o}^{-}}, V_{\mathrm{Te}+}, V_{\mathrm{Te}}\right)$ não foram afetados pelo incremento na ingestão de P. Apesar do efeito dos tratamentos sobre a retenção e reabsorção de $\mathrm{P}$ nos ossos e tecidos, não houve efeito $(\mathrm{P}>0,05)$ sobre a retenção de $\mathrm{P}$ (balanço de P) nesses locais (Tab. 4). 
Tabela 5. Valores médios dos fluxos de fósforo usando-se o modelo Vitti et al. (2000) e Lopes et al. (2001) em ovinos suplementados com diferentes quantidades de $\mathrm{P}$

\begin{tabular}{lcccccc}
\multirow{2}{*}{ Modelo } & \multicolumn{7}{c}{ Fluxo de fósforo entre compartimentos } \\
\cline { 2 - 7 } & $F_{21}$ & $F_{12}$ & $F_{32}$ & $F_{23}$ & $F_{42}$ & $F_{24}$ \\
\hline Modelo 1 $^{*}$ (gP/dia) & $V_{\mathrm{aT}}$ & $V_{\mathrm{IT}}$ & $V_{\mathrm{o}^{+}}$ & $V_{\mathrm{o}^{-}}$ & $V_{\mathrm{Te}^{+}}$ & $V_{\mathrm{Te}^{+}}$ \\
Modelo 2 $^{* *}$ (gP/dia) & $5,27 \mathrm{a}$ & $4,54 \mathrm{a}$ & $6,55 \mathrm{a}$ & $6,74 \mathrm{a}$ & $1,76 \mathrm{a}$ & $0,96 \mathrm{a}$ \\
& $5,01 \mathrm{a}$ & $4,31 \mathrm{a}$ & $6,28 \mathrm{a}$ & $5,80 \mathrm{a}$ & $0,36 \mathrm{~b}$ & $0,34 \mathrm{~b}$
\end{tabular}

Médias seguidas de letras distintas na coluna diferem segundo o teste $\mathrm{t}(\mathrm{P}<0,05)$.

*Modelo de Vitti et al. (2000).

** Modelo de Lopes et al. (2001).

Quando comparados os valores absolutos das médias dos dois modelos (Tab. 4), para cada fluxo, não houve diferença nos fluxos de plasma para ossos e vice-versa, e sim para os valores dos fluxos equivalentes entre plasma e tecidos moles $\left(F_{42}\right.$ e $\left.F_{24}\right)$, porém o modelo 1 parece ser mais preciso na determinação desse fluxo de $\mathrm{P}$, considerando-se os resultados de Dias et al. (2007) com ovinos em crescimento e a atividade metabólica de órgãos como fígado e rins. Contudo, essas médias devem ser observadas com cautela, porque, entre tratamentos, essas variáveis apresentaram efeitos diferenciados. As diferenças poderiam ser devido ao fato de que o modelo de Vitti et al. (2000) é contemplado com um número menor de variáveis, mas todos os fluxos baseiam-se nas atividades específicas, e o modelo de Lopes et al. (2001) explica, mais detalhadamente, o fluxo e refluxos nos ossos e tecidos. Entretanto, uma parte das suas equações é derivada das diferenças entre fluxo, caracterizando este modelo como o mais empírico (Vitti et al., 2006). O modelo de Vitti et al. (2000) tem sido utilizado, também, em suínos, e os resultados parecem explicar bem o metabolismo do mineral nessa espécie, como demonstrado por Moreira et al. (2009).

\section{CONCLUSÕES}

Os níveis de ingestão de $\mathrm{P}$ avaliados interferiram no fluxo biológico de fósforo em ovinos. A disponibilidade biológica do fósforo diminuiu quando a ingestão superou a necessidade do animal, aumentando as perdas no ambiente. As diferenças entre os dois modelos podem ser atribuídas à estrutura matemática deles.

\section{AGRADECIMENTOS}

À CAPES, pela bolsa concedida (Bolsista da CAPES/CNPq - IEL Nacional - Brasil) e à FAPEMIG, pelo apoio financeiro ao Projeto (CVZ-APQ-2697.5.04/07).

\section{REFERÊNCIAS BIBLIOGRÁFICAS}

BRAITHWAITE, G.D. Studies on the absorption and retention of calcium and phosphorus by young and mature Ca-deficient sheep. Br. J. Nutr., v.34, p.311-324, 1975.

BRAVO, D.; SAUVANT, D.; BOGAERT, C. et al. II. Quantitative aspects of phosphorus absorption in ruminants. Reprod. Nutr. Dev., v.43, p.285-300, 2003.

DIAS, R.S.; KEBREAB, E.; VITTI, D.M.S.S. et al. A revised model for studying phosphorus and calcium kinetics in growing sheep. J. Anim. Sci., v.84, p.2787-2794, 2006.

DIAS, R.S.; KEBREAB, E.; VITTI, D.M.S.S. et al. Phosphorus kinetics in lambs fed different levels of dicalcium phosphate. J. Agric. Sci., v.145, p.509-516, 2007.

FERNANDEZ, J.A. Calcium and phosphorus metabolism in growing pigs: a model resolution. III. Livest. Prod. Sci., v.41, p.255-261, 1995.

FISKE, C.H.; SUBBAROW, Y. The colorimetric determination of phosphorus. J. Biol. Chem., v.66, p.375-400, 1925.

KLEIBER, M.; SMITH, A.H.; RALSTON, N.P. et al. Radiophosphorus $\left(\mathrm{P}^{32}\right)$ as tracer for measuring endogenous phosphorus in cows feces. J. Nutr., v.45, p.253-263, 1951. 
LOPES, J.B.; VITTI, D.M.S.S.; ABDALLA, A.L. et al. Modelo de fluxo biológico do fósforo de fontes de fosfato em suínos, usando $0{ }^{32} \mathrm{P}$ como marcador. Rev. Bras. Zootec., v.30, p.165173, 2001.

LOUVANDINI, H.; VITTI, D.M.S.S. Cinética de fósforo com modelos matemáticos em ovinos adultos. Pesq. Agrop. Bras., v.42, p.1467-1472, 2007.

MOREIRA, J.A.; LOPES, J.B.; VITTI, D.M.S.S. et al. Modelos matemáticos para o estudo do fluxo biológico de fósforo em suínos alimentados com dietas suplementadas com níveis crescentes de fitase. Arq. Bras. Med. Vet. Zootec., v.61, p.420-428, 2009.

NASCIMENTO FILHO, V.F.; LOBÃO, A.O. Detecção de P-32 em amostras de origem animal e vegetal por efeito Cerenkov, cintilação líquida e detector GM. Piracicaba: CENA, 1977. 25p. (Boletim Científico, 48)

NUTRIENT requirements of small ruminants: sheep, goats, cervids, and new word camelids. Washington: National Academic, 2007. 362p.

OFFICIAL methods of analysis. 16.ed. Washington: AOAC, 1995.

PORTILHO, F. P.; VITTI, D. M. S. S.; ABDALLA, A. L. et al. Minimum phosphorus requirement for Santa Inês lambs reared under tropical conditions. Small Rum. Res., v.63, p. 170-176, 2006.
SARRUGE, J.R.; HAAG, H.P. Análises químicas em plantas. Piracicaba: ESALQ/USP, 1974. 56p.

STATISTICAL package for the social sciences. Release 15.0 Chicago, Ill: Editora, 2006.

VAN SOEST, P.J.; WINE, R.H. Use of detergent in the analysis of farmers feed. IV. Determination of plant cell wall constituents. $J$. Assoc. Anal. Chem., v.50, p.50-55, 1967.

VITTI, D.M.S.S.; KEBREAD, E.; ABDALLA, A.L. et al. A kinetic models of phosphorus metabolism in growing goats. J. Anim. Sci., v.78, p.2706-2716, 2000

VITTI, D.M.S.S.; ROQUE, A.P.; DIAS, R. et al. Metabolismo de cálcio em ovinos em crescimento sob suplementação com diferentes fontes de cálcio: aplicação e comparação de dois modelos matemáticos. Rev. Bras. Zootec., v.35, p.2487-2495, 2006.

ZAGATTO, E.A.G.; KRUG, F.J.; BERGAMIN FILHO, H. et al. Merging zones in flow injection analysis. 2. Determination of calcium, magnesium and potassium in plant material by flow injection atomic absorption and flame emission spectrometry. Anal. Chem. Acta, v.104, p.279-284, 1979. 